\title{
Clinical Validation of the Spirohome Clinic Ultrasonic Spirometer in Child and Adolescent Patients
}

\author{
Bulent Enis Sekerel', Hilal Unsal', Umit Murat Sahiner', Ozge Uysal Soyer', Ebru Damadoglu², \\ Gul Karakaya ${ }^{2}$, Ali Fuat Kalyoncu ${ }^{2}$ \\ 'Division Pediatric Allergy and Asthma, Hacettepe University Faculty of Medicine, Ankara, Turkey; ${ }^{2}$ Division of Allergy and Immunology, Department \\ of Chest Diseases, Hacettepe University Faculty of Medicine, Ankara, Turkey \\ Correspondence: Bulent Enis Sekerel, Tel +90 312305 17 00, Fax +90 31230550 00, Email bsekerel@hacettepe.edu.tr
}

Background: Spirometers are critical devices that reveal the respiratory dynamics caused by respiratory problems and their severity and facilitate their diagnosis and follow-up. Hand-held spirometers have emerged relatively recently and offer several advantages over conventional desktop systems. There remains, however, a need for reassurance of high-quality spirometry testing with next-generation portable spirometers that connect over Bluetooth ${ }^{\circledR}$ to smart device applications. In this study, we examine the accuracy and repeatability of lung function measurements of a novel hand-held ultrasonic spirometer, the Spirohome Clinic and compare its clinical performance to a reference device, the EasyOne Air.

Methods: Benchtop validation of the spirometers was conducted using a lung simulator device according to ATS/ERS guidelines and the ISO 26782 standard waveforms. Subsequently, 48 volunteers (pediatric patients between 6 and 11 years of age and adolescent patients between 12 and 18 years of age) performed spirometry with both the Spirohome Clinic and the EasyOne Air spirometer during their clinic visits. Spirometric data including repeated $\mathrm{FEV}_{1}, \mathrm{FVC}, \mathrm{FEV}_{6}, \mathrm{FEF}_{25-75}$, and PEF measurements were collected.

Results: Both the Spirohome Clinic and the EasyOne Air successfully passed requirements for accuracy stated in relevant guidelines and standards for spirometry. The only statistically significant $(p<0.05)$ difference was for FVC measurement accuracy. Clinical comparisons revealed strong correlation between spirometers in the measurement of key pulmonary function parameters including $\mathrm{FEV}_{1}$ and FVC with a Pearson's correlation coefficient of 0.99. Bland-Altman plots showed good agreement between mean differences of $\mathrm{FEV}_{1}$ and $\mathrm{FVC}$ with the majority measurements remaining between the limits of $95 \%$ agreement for both the entire patient cohort and also in age and gender subsets.

Conclusion: The present study demonstrated that the Spirohome Clinic spirometer conforms to ATS/ERS performance requirements and validates the clinical comparability of its measurement accuracy and repeatability to the EasyOne Air. These findings support the indicated use of the Spirohome Clinic for high-quality lung function testing in clinical settings.

Keywords: handheld spirometer, ultrasonic spirometer, portable spirometer, spirometry, pediatric, respiratory disease, FEV1, FVC

\section{Introduction}

Lung diseases are among the most common non-communicable medical conditions in the world. A study conducted between 1990 and 2017 showed that chronic respiratory diseases increased by $39.8 \%$, and close to 545 million people had diseases in the world. ${ }^{1}$ Spirometry remains the "gold standard" for assessing obstructive and/or restrictive airway diseases ${ }^{2-4}$ in both adults and children. ${ }^{5}$ Spirometers are reliable and non-invasive tools that can characterize flow in both the large and small airways. They can be categorized technologically based on the type of flow sensor they contain. Although pneumotachometer-based ${ }^{6}$ and turbine-based ${ }^{7}$ spirometers are still used in clinical spirometry, current state-ofthe-art spirometers utilize ultrasound-based flow sensors. Ultrasonic spirometers have become renowned for their accuracy, and their sensitivity to lower flow rates. Unlike turbine-based spirometers, ultrasonic spirometers have no moving parts and are thus low-maintenance devices that do not require routine re-calibration. Turbine-based spirometers 
also have difficulty reaching low flow sensitivity which is limiting in conditions like COPD where patients have extended periods of low flow exhalation in spirometry tests.

Spirometers are also classified by whether they are conventional desktop systems or hand-held devices. Hand-held spirometers offer portability, are generally space-saving and often less expensive than conventional desktop spirometers. Today, hand-held spirometers are designed with features like on-screen guidance, real-time feedback and session grading which aim to reduce the need for expert intervention and boost user confidence during spirometry sessions. With the rapid growth of digital health technologies and remote care through telehealth platforms, integratable patient-centric smart spirometers have become increasingly popular in the pulmonary health field. These modern spirometers are not only proving to help solve the underutilization problem seen with traditional spirometers in the clinic, but they are moving lung function testing to home settings where patients are liberated from the burden and costs of travel whilst still being able to provide lung function data to their health-care professionals for monitoring. Such technologies have become particularly relevant in recent times as the COVID-19 pandemic has led to the closures of pulmonary function laboratories around the world to reduce patient and personnel exposure to the virus. However, hand-held devices are still establishing their reliability in clinical spirometry as full-scale spirometers.

Due to their relative newness, validation of hand-held spirometers in comprehensive lung function testing remains a point of interest for the respiratory health community. There are several examples in literature comparing hand-held and conventional spirometers ${ }^{8-15}$ and with each other. ${ }^{16-18}$ The advent of spirometry platforms that combine portable spirometers with applications running on smart devices raises further concerns such as those regarding the device's processing power, interface components, robustness, and reproducibility of its measurements. ${ }^{14}$ Whilst portable spirometry platforms aim to and seemingly achieve making spirometry more readily accessible and appealing to professionals and patients, it is still critical that they are rigorously validated through clinical validation studies.

This work discloses a two-phase validation study of the Spirohome Clinic ultrasonic spirometer using the EasyOne Air device as a contrast. First, devices were tested in the laboratory to assess compliance to the accuracy and performance criteria detailed in relevant ATS/ERS guidelines and the ISO 26782 standard. Then, a group of pediatric patients with pulmonary symptoms from Pediatric Allergy and Asthma at Hacettepe University Hospital who met the inclusion criteria performed pulmonary function tests with both devices during clinical visits. The accuracy and repeatability of certain spirometric parameters from both devices were then statistically compared.

\section{Materials and Methods}

\section{Lung Simulator Analysis}

The accuracy of both spirometers was assessed with a lung simulator as suggested in the ATS guideline and described in the BS EN ISO 26782 document. The spirometric parameters FEV 1 (L), FVC (L), and PEF (L/s) were measured thrice each with the Spirohome Clinic and EasyOne under a set of 13 different waveforms created by the PWG-33 lung simulator (Piston Medical, Budapest, Hungary). The repeatability (L) and accuracy (L) of the spirometric parameters measured by each device were calculated. Repeatability was calculated as the difference between the best and secondbest measurements, and accuracy was the difference between the mean of three measurements and the target value (the target value is calculated by the lung simulator device).

\section{Clinical Pulmonary Function Tests}

The pulmonary function tests of participants were conducted with both the Spirohome Clinic and the EasyOne Air spirometers. EasyOne Air was chosen as a reference device due to its similarity in flow sensor technology and its history of clinical application. ${ }^{19,20}$ Both spirometers were factory-calibrated, however, performance accuracy was also confirmed with laboratory lung simulator tests before participant use. Participants were required to perform at least 3 spirometry trials (maximum limit of 8) with each device with a 30-minute quiescent period between each instrument. Of the 48 participants that presented to the clinic, the first 24 used the Spirohome Clinic first and the EasyOne Air second, and this order was reversed for the next 24 patients to prevent potential bias. All measurements were guided in a standardized way by the same trained personnel in accordance with current ATS/ERS guidelines. 
$\mathrm{FEV}_{1}, \mathrm{FVC}, \mathrm{FEV}_{6}, \mathrm{FEF}_{25-75}$, and PEF values obtained from each spirometer was recorded digitally for subsequent statistical analysis. The acceptability of $\mathrm{FEV}_{1}$ and $\mathrm{FVC}$ measurements was assessed according to the ATS guideline grading system. ${ }^{21}$ To ensure consistency and validity of data, the results ranked as either a grade A or B for $\mathrm{FEV}_{1}$ and FVC measurements were included in the study.

\section{Study Design and Population}

The present work presents a cross-sectional, prospective, single-center comparative study. The intended patient sample size was calculated with a priori power analysis performed based on $\mathrm{FEV}_{1}$ and $\mathrm{FVC}$ measurements. Based on the power analysis and with reference to methods reported in a similar study, ${ }^{15}$ a total of 48 participants were required for detecting a mean difference of $0.25 \mathrm{~L}$ between the spirometers to achieve $80 \%$ statistical power at $\alpha=0.05$, when the standard deviation is $0.6 \mathrm{~L}$ for FEV1 and FVC.

Healthy controls and individuals with asthma or allergic rhinitis presenting with a cough that were between the ages of 6-18 (pediatric patients between 6 and 11 years of age and adolescent patients between 12 and 18 years of age) and who previously demonstrated ability in performing spirometry tests were included in the study. Patients were selected from those presenting to the Hacettepe University Pediatric Allergy and Asthma outpatient clinic with asthma, allergic rhinitis, or another condition and who volunteered to take part in the study.

Participants (patients and parents/legal guardians) were provided with information regarding the purpose, content and significance of the study and written consent forms were collected (from both patients and parents/legal guardians) prior to enrolment. Participant exclusion criteria were as follows: history of syncope due to difficult expiration or cough, having cardiac arrhythmia, history of brain, thoracic or abdominal surgery in the last four weeks, eye, sinus, or middle ear surgery in the last week, presence of pneumothorax, uncompensated heart failure, cerebral aneurysms, hemoptysis, significant secretion, oral lesion, oral bleeding, nausea, vomiting, unconsciousness, dementia, muscle disease or bone disease and respiratory or systemic infections.

Patients meeting inclusion criteria and presenting to the clinic within a specified date range were evaluated by an experienced pediatrician. Participant eligibility was also dependent on their capacity to comply with the requirements of performing a correct pulmonary function test. Only participants who consented to participate were included in the study and only a couple of patients declined participation in the study due to time constraints. The study commenced on 12 August 2020 and concluded on 18 December 2020.

\section{Ethics Approval and Consent to Participate}

Ethics approval to conduct this study was obtained from the Ethics Board of Approval at Hacettepe University (Decision Number: 2020/06-39 (KA-20037) and the Turkish Ministry of Health (Decision Number: 68869993-511.06-E.148267)). Informed written consent was also obtained from each participant before inclusion in the study.

\section{Spirometers}

The two spirometers comparatively assessed in this study were the Spirohome Clinic (Inofab Health, Ankara, Turkey) and EasyOne Air (ndd Medizintechnik AG, Zurich, Switzerland). The Spirohome Clinic is a CE-certified ultra-portable ultrasonic spirometer that pairs via Bluetooth ${ }^{\circledR}$ to smart tablets and desktop computers running the accompanying application. The following indices obtained from spirometry are stored through the application: forced vital capacity $(\mathrm{FVC})$, forced expiratory volume in $1 \mathrm{~s}\left(\mathrm{FEV}_{1}\right), \mathrm{FEV}_{1} / \mathrm{FVC}$ ratio, forced expiratory volume in $6 \mathrm{~s}\left(\mathrm{FEV}_{6}\right)$, peak expiratory flow (PEF), forced expiratory flow at $25-75 \%\left(\mathrm{FEF}_{25-75}\right)$ of forced vital capacity, back extrapolated volume (BEV) and Forced expiratory time (FET). The Spirohome Clinic also integrates to an accompanying cloud platform and local electronic health record systems to facilitate telehealth-based pulmonary care and scaled respiratory research. The EasyOne Air is a CE-certified and FDA-cleared ultrasonic spirometer that has an on-screen display. It can be used as a portable or as a PC spirometer. It produces the same set of spirometric data as the Spirohome Clinic. Table 1 shows key differences and similarities between the SpiroHome Clinic and the Easyone Air features. 
Table I SpiroHome Clinic and EasyOne Air Features

\begin{tabular}{|c|c|c|}
\hline Feature & SpiroHome Clinic & NDD EasyOne Air \\
\hline Sensor Type & Ultrasonic & Ultrasonic \\
\hline Display Interface & iOS/Android Tablets, PC & On-screen display, PC \\
\hline Test Storage Capacity & Unlimited (Cloud database) & Up to 10,000 tests \\
\hline Mouthpiece Accessory & SpiroWay Reusable Mouthpiece & EasyOne Flow Tube \\
\hline Volume Accuracy & $\pm 2 \%$ or $0.05 \mathrm{~L}$ & $\pm 2 \%$ or $0.05 \mathrm{~L}$ \\
\hline Flow Accuracy & $<\% 2$ & $\pm 2 \%$ or $0.02 \mathrm{~L} / \mathrm{s}$ \\
\hline ATS 2019 Compliancy & Yes & Yes \\
\hline Predicted Values & $\begin{array}{l}\text { Quanjer } 2012 \text { (GLI), NHANES III } 1999 \text { (Hankinson), ERS } \\
1993 \text { (ECCS, EGKS, Quanjer), Crapo 198I, Knudson } 1976\end{array}$ & $\begin{array}{l}\text { ERS (ECCS), Roca, Austria, NHANES III, Knudson 1976, } \\
\text { Knudson 1983, Crapo, Morris, Cherniak }\end{array}$ \\
\hline Parameters & $\begin{array}{l}\text { FVC, FEV0.75, FEVI, FEV3, FEV6, FEV0.75/FVC, FEVI/FVC, } \\
\text { FEV3/FVC, FEV6/FVC, PEF, MMEF, FEF25, FEF25-75, MET25- } \\
\text { 75, FEV0.75/FEV6, FEVI/FEV6, FEEF50/FVC, MMEF/FVC, } \\
\text { FET, BEV, FIFI, FIVC, PIF, FIF25-75, FIVI/FIVC, R50 (FEF50/ } \\
\text { FIF50), VC, VCin, VCex, ERV, IRV, IC, Rf, VT, MVV, MVV6, } \\
\text { MVVtime }\end{array}$ & $\begin{array}{l}\text { FEVI, FVC, FEVI/FVC, FEV6, FEVI/FEV6, MEF25-75, MEF25, } \\
\text { MEF50, MEF75, PEF, FET, FIVC, PIF, IVC, IRV, ERV, FEVI/VC, } \\
\text { MVV, pre-post \% variation, QC rating }\end{array}$ \\
\hline Graphical Output & Volume/Time, Flow /Volume (exhalation \& inhalation loops) & Volume/Time, Flow /Volume (exhalation \& inhalation loops) \\
\hline Session Grading & Yes & Yes \\
\hline ATS Error Codes Displayed & $\begin{array}{l}\text { Hesitation, Plateau, Cough, Early Termination, Late Peak } \\
\text { Flow }\end{array}$ & $\begin{array}{l}\text { Hesitation, Plateau, Cough, Early Termination, Late Peak } \\
\text { Flow }\end{array}$ \\
\hline $\begin{array}{l}\text { Personal Spirometer } \\
\text { Integration }\end{array}$ & Yes & No \\
\hline
\end{tabular}

\section{Demographics and Anthropometrics}

Participants assessed and approved by the pediatrician for inclusion in the study were taken to a designated room to perform the lung function tests. The age and gender of all participants were noted, and their weight and height were measured. No other demographic or anthropometric data was collected.

\section{Statistical Analysis}

The mean \pm SD and median (Interquartile Range) repeatability and accuracy values for spirometric data recorded over the 13 different waveforms produced by the lung simulator are given in both liters and as a percent ratio. The repeatability and accuracy of spirometric data were statistically compared between the Spirohome Clinic and reference device.

Correlation between spirometers was also assessed with correlation plots and the Pearson's correlation coefficient. A simple linear regression line was fitted to facilitate comparisons. Bland-Altman plots and paired $t$-tests were 
Table 2 Lung Simulator Analysis Results. Differences in Repeatability and Accuracy Measures Between Spirohome and EasyOne Air

\begin{tabular}{|c|c|c|c|c|c|c|}
\hline & \multicolumn{2}{|c|}{ FEV I (L) } & \multicolumn{2}{|c|}{ FVC (L) } & \multicolumn{2}{|c|}{ PEF (L/s) } \\
\hline & Spirohome & EasyOne & Spirohome & EasyOne & Spirohome & EasyOne \\
\hline \multicolumn{7}{|l|}{ Repeatability (L) } \\
\hline Mean $\pm S D$ & $0.00 \pm 0.01$ & $0.00 \pm 0.01$ & $0.00 \pm 0.01$ & $0.00 \pm 0.02$ & $0.00 \pm 0.02$ & $0.00 \pm 0.02$ \\
\hline Median (IQR) & $0.00(0.01)$ & $0.00(0.01)$ & $-0.01(0.01)$ & $0.00(0.01)$ & $0.00(0.03)$ & $-0.01(0.03)$ \\
\hline $\mathrm{p}$-value & \multicolumn{2}{|c|}{0.264} & \multicolumn{2}{|c|}{0.448} & \multicolumn{2}{|c|}{0.264} \\
\hline \multicolumn{7}{|l|}{ Repeatability (\%) } \\
\hline Mean $\pm S D$ & $-0.05 \pm 0.19$ & $0.10 \pm 0.31$ & $-0.14 \pm 0.24$ & $0.11 \pm 0.58$ & $-0.09 \pm 0.55$ & $-0.28 \pm 0.48$ \\
\hline Median (IQR) & $0.00(0.21)$ & $0.00(0.24)$ & $-0.16(0.27)$ & $0.00(0.14)$ & $0.00(0.62)$ & $-0.25(0.80)$ \\
\hline $\mathrm{P}$-value & \multicolumn{2}{|c|}{0.264} & \multicolumn{2}{|c|}{0.311} & \multicolumn{2}{|c|}{0.223} \\
\hline \multicolumn{7}{|l|}{ Accuracy (L) } \\
\hline Mean $\pm S D$ & $0.01 \pm 0.02$ & $0.01 \pm 0.02$ & $0.03 \pm 0.03$ & $-0.02 \pm 0.04$ & $0.02 \pm 0.07$ & $0.02 \pm 0.11$ \\
\hline Median (IQR) & $0.00(0.04)$ & $0.00(0.03)$ & $0.03(0.03)$ & $-0.01(0.04)$ & $0.03(0.09)$ & $0.00(0.16)$ \\
\hline $\mathrm{p}$-value & \multicolumn{2}{|c|}{0.264} & \multicolumn{2}{|c|}{$0.001 *$} & \multicolumn{2}{|c|}{0.724} \\
\hline \multicolumn{7}{|l|}{ Accuracy (\%) } \\
\hline Mean $\pm S D$ & $0.19 \pm 0.54$ & $-0.13 \pm 0.64$ & $0.47 \pm 0.5$ & $-0.89 \pm 1.48$ & $2.63 \pm 5.79$ & $1.29 \pm 2.97$ \\
\hline Median (IQR) & $0.14(1.00)$ & $0.00(1.28)$ & $0.53(0.15)$ & $-0.51(1.7)$ & I.34 (2.80) & $0.29(4.45)$ \\
\hline $\mathrm{p}$-value & \multicolumn{2}{|c|}{0.204} & \multicolumn{2}{|c|}{$<0.001 *$} & \multicolumn{2}{|c|}{0.614} \\
\hline
\end{tabular}

Notes: Repeatability (L): the difference between best and second-best trials. Repeatability (\%): Repeatability/Target*100. Accuracy (L): Mean of three measurements - target value. *Indicates statistical significance at $\mathrm{p}<0.05$.

Abbreviations: Accuracy (\%), Accuracy/Target*100; diff, difference; IQR, Interquartile range; P-values, P-values are obtained with the Mann-Whitney U-test.

applied to depict bias between mean differences for spirometric parameters recorded by each device. Finally, Kruskal Wallis and post-hoc Dunn's tests were used to compare error rates between spirometers. The results were evaluated within a 95\% confidence interval and at a level of $p<0.05$ significance. Spirometric data were also analyzed in age and gender subgroups. All statistical analyses were performed using R version 4.0.3 (R Development Core Team, 2010; www.R-project.org) and run in RStudio (www.rstudio.com). Figures were generated by using the ggplot2 package in $R$.

\section{Results}

\section{Lung Simulator Analysis Results}

The mean and median accuracy and repeatability values for $\mathrm{FEV}_{1}, \mathrm{FVC}$ and PEF measurements from the 13 different test waveforms collected by each spirometer are given in Table 2 (please see the Supplementary Information for details). Values are presented both in liters (L) or liter per second (L/s) and percent (\%). Both spirometers met ATS limits for measurement accuracy in all 13 ISO 26782 waveforms tested. There was a statistically significant $(p<0.05)$ difference between spirometers with respect to FVC accuracy both in units of liters and percentage. The SpiroHome Clinic mean FVC accuracy value $(0.03 \pm 0.03 \mathrm{~L})$ remains at the upper limit for accuracy as defined by the relevant standard, and the EasyOne Air mean FVC accuracy value $(-0.02 \pm 0.04 \mathrm{~L})$ remains at the lower limit for accuracy.

\section{Study Population and Pulmonary Function Results}

The study group consisted of 48 persons between the ages of 6-18 years. The mean age of the study population was 12.7 \pm 3.5 years. Thirteen $(27.1 \%)$ of the participants were female. Other demographics and pulmonary function test results for both spirometers are presented in Table 3. Results were also presented separately for two age groups, children (6-11 years old) and adolescents (12-18 years old). 
Table 3 Study Population Characteristics

\begin{tabular}{|c|c|c|c|}
\hline & \multirow[b]{2}{*}{ Total $(n=48)$} & \multicolumn{2}{|c|}{ Age Group } \\
\hline & & $6-11$ Years $(n=18)$ & $12-18$ Years $(n=30)$ \\
\hline \multicolumn{4}{|l|}{ Gender } \\
\hline Male & 35 (72.9) & $13(72.2)$ & $22(73.3)$ \\
\hline Female & $13(27.1)$ & $5(27.8)$ & $8(26.7)$ \\
\hline Age (years) & $12.7 \pm 3.5$ & $8.8 \pm 1.4$ & $|5| \pm 2.0$. \\
\hline Weight (kg) & $56.0 \pm 20.0$ & $39.8 \pm 14.8$ & $65.7 \pm 16.1$ \\
\hline Height (cm) & $156 \pm 18.4$ & $138 \pm 10.7$ & $167 \pm 11.9$ \\
\hline FEV , Spirohome(L) & $2.63 \pm 0.95$ & $1.79 \pm 0.48$ & $3.13 \pm 0.80$ \\
\hline FEV, EasyOne Air(L) & $2.56 \pm 0.94$ & $1.73 \pm 0.43$ & $3.06 \pm 0.80$ \\
\hline FVC Spirohome(L) & $3.30 \pm 1.21$ & $2.20 \pm 0.62$ & $3.97 \pm 0.97$ \\
\hline FVC EasyOne Air(L) & $3.24 \pm 1.19$ & $2.13 \pm 0.57$ & $3.91 \pm 0.93$ \\
\hline FEV $_{6}$ Spirohome(L) & $3.29 \pm 1.21$ & $2.19 \pm 0.62$ & $3.95 \pm 0.98$ \\
\hline $\mathrm{FEV}_{6}$ EasyOne Air(L) & $3.23 \pm 1.19$ & $2.12 \pm 0.55$ & $3.90 \pm 0.94$ \\
\hline $\mathrm{FEF}_{25-75}$ Spirohome(L) & $2.48 \pm 1.03$ & $1.79 \pm 0.72$ & $2.90 \pm 0.98$ \\
\hline $\mathrm{FEF}_{25-75}$ EasyOne Air(L) & $2.44 \pm 1.04$ & $1.79 \pm 0.70$ & $2.83 \pm 1.02$ \\
\hline PEF Spirohome(L) & $5.89 \pm 1.89$ & $4.26 \pm 0.97$ & $6.87 \pm 1.61$ \\
\hline PEF EasyOne Air(L) & $5.57 \pm 1.87$ & $3.94 \pm 0.93$ & $6.54 \pm 1.59$ \\
\hline
\end{tabular}

Notes: Values are presented as $\mathrm{n}(\%)$ or mean \pm SD. A detailed comparison of the FEV and FVC measurement results of the two devices according to gender and age was presented in Table 4. Overall, the mean difference was statistically significant $(p<0.05)$ for both FEV 1 and FVC. Consistently higher measurements of the Spirohome Clinic for all parameters compared to the EasyOne Air is likely to have contributed to this finding. Subset analysis showed that the difference of spirometers for both FEV 1 and FVC was statistically significant $(p<0.05)$ for males and participants in the $12-18$ year age bracket.

Table 4 Mean Differences (with 95\% Confidence Intervals) in FEV $(\mathrm{L})$ and FVC (L) Measurements Between Spirohome and EasyOne Across Gender and Age Subgroups

\begin{tabular}{|c|c|c|c|c|c|}
\hline & \multirow[b]{2}{*}{ Overall $(n=48)$} & \multicolumn{2}{|l|}{ Gender } & \multicolumn{2}{|l|}{ Age Group } \\
\hline & & Males $(n=35)$ & Female $(n=13)$ & $6-11$ Years $(n=18)$ & $12-18$ Years $(n=30)$ \\
\hline \multicolumn{6}{|l|}{$\mathrm{FEV}_{1}(\mathrm{~L})$} \\
\hline Mean diff. (L) & $0.065^{*}$ & $0.082^{*}$ & 0.021 & 0.064 & $0.066^{*}$ \\
\hline $95 \% \mathrm{Cl}$ & $(0.024,0.107)$ & $(0.029,0.135)$ & $(-0.036,0.078)$ & $(-0.005,0.133)$ & $(0.012,0.121)$ \\
\hline \multicolumn{6}{|l|}{$\mathrm{FVC}(\mathrm{L})$} \\
\hline Mean diff. (L) & $0.059 *$ & $0.08 I^{*}$ & -0.001 & 0.069 & $0.053^{*}$ \\
\hline $95 \% \mathrm{Cl}$ & $(0.015,0.102)$ & $(0.024,0.138)$ & $(-0.047,0.045)$ & $(-0.022,0.161)$ & $(0.005,0.100)$ \\
\hline
\end{tabular}

Note: $*_{p}<0.05$ based on paired $t$-test.

Abbreviations: Mean difference, Spirohome-EasyOne; diff, difference; $\mathrm{Cl}$, confidence interval.

$\mathrm{FEV}_{6}, \mathrm{FEF}_{25-75}$, and PEF measurements obtained with the Spirohome spirometer were higher than that of the EasyOne spirometer (Table 5). Significant differences in $\mathrm{FEV}_{6}$ and PEF measurements between the two spirometers were observed in males and 12-18 years old volunteers. There was a larger difference in mean $\mathrm{FEF}_{25-75}$ volume recorded by each spirometer in patients over the age of 12 and it was also larger in males compared to females.

Correlation analysis of $\mathrm{FEV}_{1}$ and FVC values $(\mathrm{n}=48)$ obtained from two spirometers is presented in Figure 1. For both values, the Pearson correlation coefficient was 0.99 , and $p$ values were $<0.001$. The simple linear regression line was fitted with $\mathrm{r}=0.99, p<0.001$ for $\mathrm{FEV}_{1}$ Spirohome and FVC Spirohome (Figure 1). The regression equations show that the Spirohome Clinic measured higher $\mathrm{FEV}_{1}$ and $\mathrm{FVC}$ values than the EasyOne Air.

In Figure 2, the agreement between the measurements ( $\mathrm{FEV}_{1}$ and $\mathrm{FVC}$ ) is shown using Bland-Altman plots. Figure 2A presents Bland-Altman plots for the differences between $\mathrm{FEV}_{1}$ and $\mathrm{FVC}$ in all volunteers. Figure 2B shows 
Table 5 Mean Differences (with 95\% Confidence Intervals) in Measurements Between Spirohome and EasyOne Air Across Gender and Age Groups

\begin{tabular}{|c|c|c|c|c|c|}
\hline & \multirow[b]{2}{*}{ Overall $(n=48)$} & \multicolumn{2}{|l|}{ Gender } & \multicolumn{2}{|l|}{ Age Group } \\
\hline & & Males $(n=35)$ & Female $(n=13)$ & $6-11$ Years $(n=18)$ & $12-18$ Years $(n=30)$ \\
\hline \multicolumn{6}{|l|}{$\mathrm{FEV}_{6}$} \\
\hline Mean diff.(L) & $0.06 I^{* *}$ & $0.086 * * *$ & -0.004 & 0.077 & $0.052^{*}$ \\
\hline $95 \% \mathrm{Cl}$ & $(0.016,0.107)$ & $(0.027,0.145)$ & $(-0.054,0.045)$ & $(-0.022,0.176)$ & $(0.005,0.100)$ \\
\hline \multicolumn{6}{|l|}{$\mathrm{FEF}_{25-75}$} \\
\hline Mean diff.(L) & 0.040 & 0.060 & -0.011 & -0.005 & 0.068 \\
\hline $95 \% \mathrm{Cl}$ & $(-0.051,0.132)$ & $(-0.058,0.179)$ & $(-0.145,0.124)$ & $(-0.154,0.144)$ & $(-0.054,0.191)$ \\
\hline \multicolumn{6}{|l|}{ PEF } \\
\hline Mean diff.(L/s) & $0.323 * * *$ & $0.37 I^{* * *}$ & 0.193 & $0.317^{* *}$ & $0.327^{* *}$ \\
\hline $95 \% \mathrm{Cl}$ & $(0.167,0.479)$ & $(0.177,0.565)$ & $(-0.079,0.465)$ & $(0.119,0.516)$ & $(0.098,0.555)$ \\
\hline
\end{tabular}

Note: $*_{p}<0.05, * * p<0.01$, and $* * * p<0.001$ based on paired $t$-test.

Abbreviations: Mean difference, Spirohome-EasyOne; diff, difference; $\mathrm{Cl}$, confidence interval.

Bland-Altman plots for differences between $\mathrm{FEV}_{1}$ and FVC based on gender subgroups. Figure 2C shows Bland-Altman plots for differences between $\mathrm{FEV}_{1}$ and $\mathrm{FVC}$ based on age subgroups. According to the Bland-Altman plots, the majority of the results were within the limits of agreement.

\section{Discussion}

The Spirohome Clinic ultrasonic spirometer (Inofab Health, Ankara, Turkey) is a hand-held spirometer that connects to a smart device application via Bluetooth ${ }^{\circledR}$ connection. The findings of this study support the accuracy and repeatability of measurements taken with the Spirohome Clinic spirometer. The results show a high agreement and concordance between the Spirohome clinic and the reference spirometer when used in a pediatric population which includes patients with different respiratory conditions and also healthy controls.
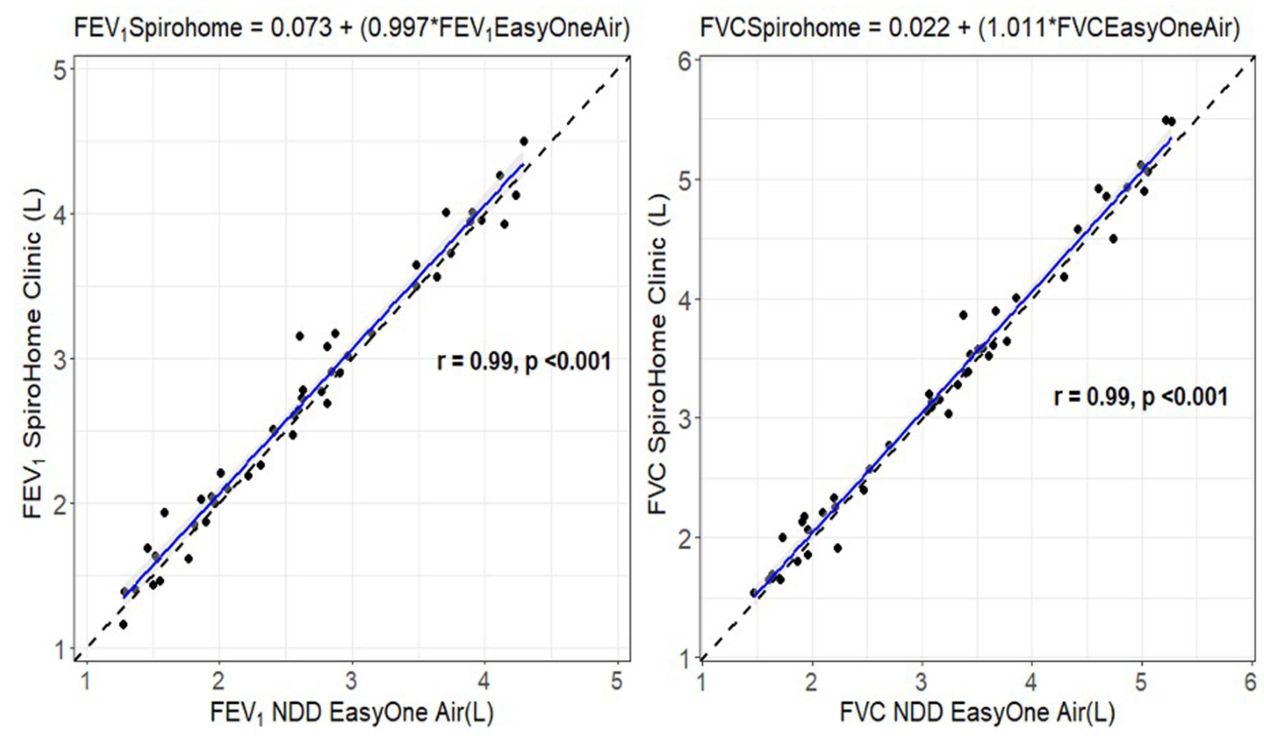

Figure I FEV, and FVC correlation analysis between spirometers. 

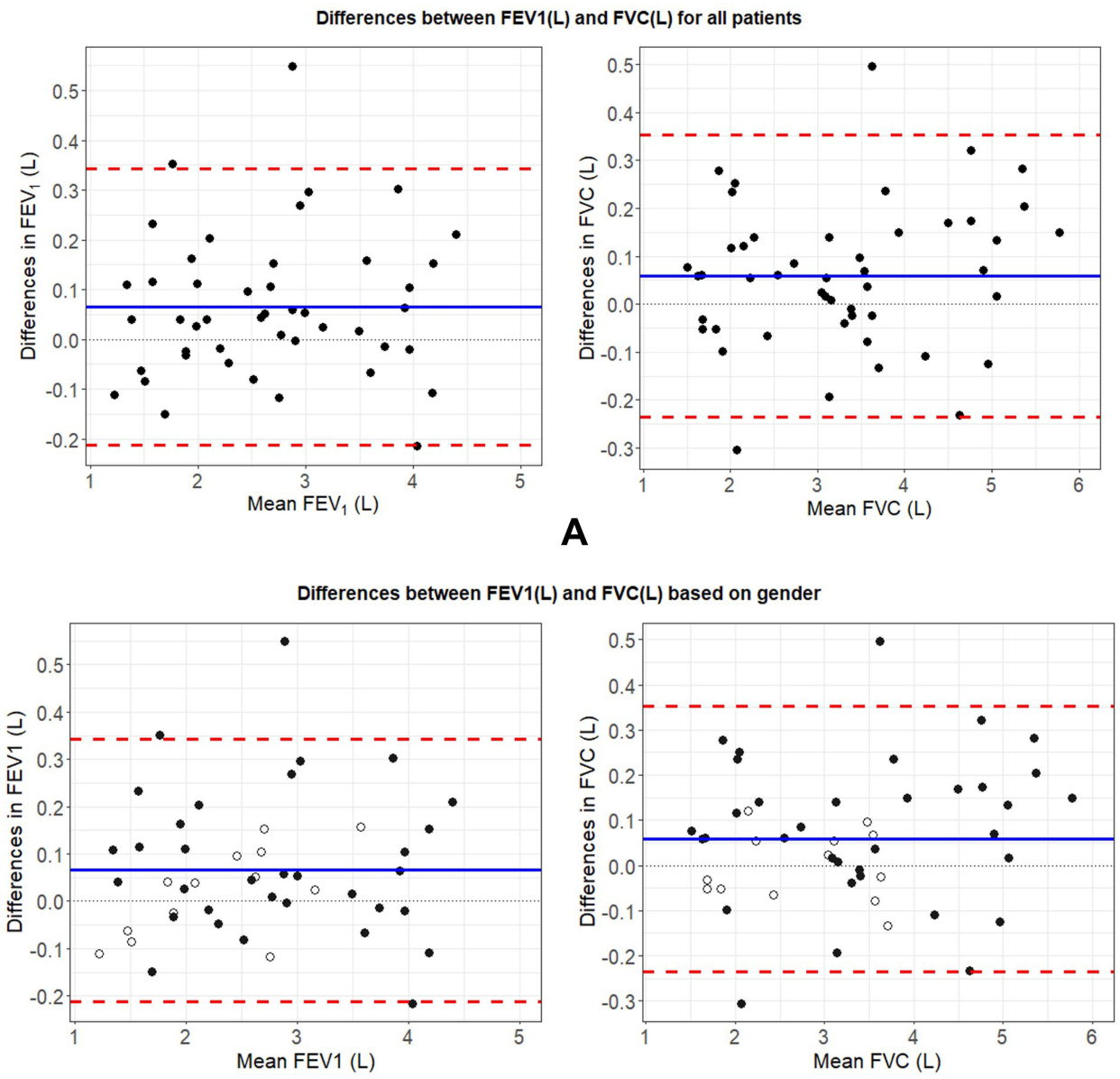

Gender $\circ$ Female - Male

B

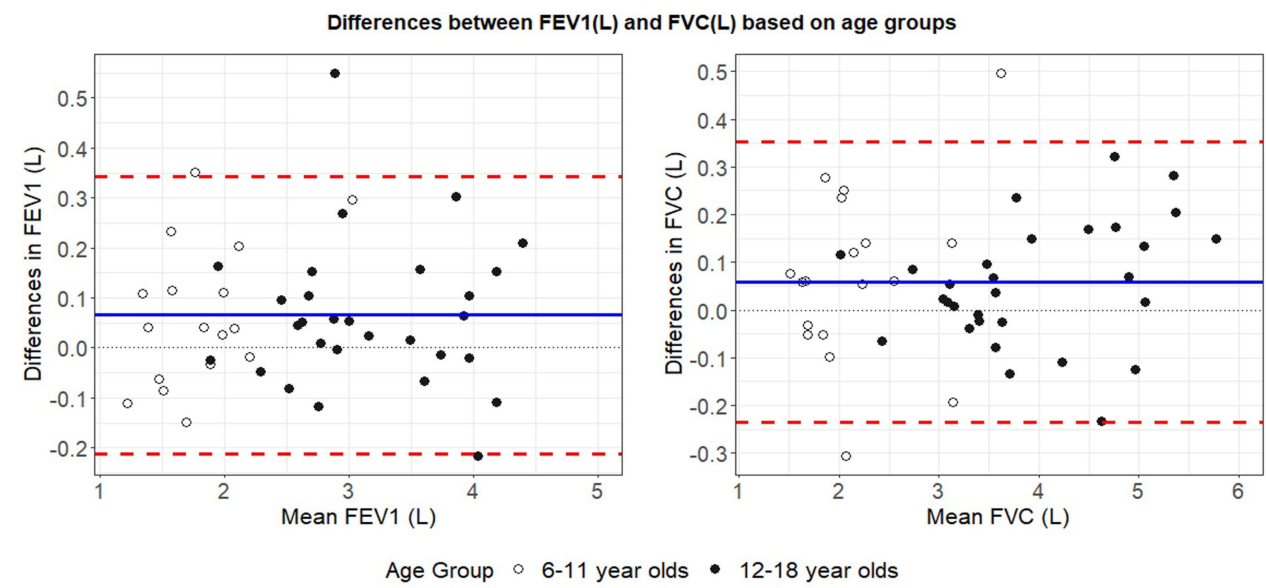

C

Figure 2 Bland-Altman plot showing agreement between FEV and FVC measurements. (A) Differences between FEVI (L) and FVC (L) for all patients. (B) Differences between FEVI (L) and FVC (L) based on gender. (C) Differences between FEVI (L) and FVC (L) based on age groups.

Lung simulator analysis is currently the only method of objectively quantifying the accuracy and repeatability of spirometric measurements collected by spirometers. The accuracy and repeatability of $\mathrm{FEV}_{1}$, FVC and PEF values recorded by the Spirohome Clinic and EasyOne Air were examined with a lung simulator according to the methods and 
waveforms given in the relevant ISO document. The Standardization of Spirometry 2019 Update published by the ATS ${ }^{21}$ states that the maximum allowed error in volume measurements in spirometers is $\pm 3.0 \%$ or $0.05 \mathrm{~L}$ (whichever is greater). Our lung simulator analyses show that the mean repeatability and accuracy errors in volume readings ( $\mathrm{FEV}_{1}$ and FVC) were less than $\pm 3.0 \%$ or $0.05 \mathrm{~L}$ in both spirometers. The only statistically significant $(p<0.05)$ difference between spirometers was found in the accuracy of the FVC, although the actual error rates were fairly small for both spirometers. We attributed the statistically significant difference to the clustering of results on different sides of zero and assume it to be a Type 1 error. Importantly, as both devices are within the accuracy limits set by the ATS, they are both deemed clinically accurate and therefore safe and effective to use in spirometry measurements. Lung simulator analysis results verified the reliability of both spirometers for further use with clinical subjects in phase two of the study.

The clinical phase of the study involved lung function testing with both devices in 48 volunteers under the age of 18 . The mean $\pm \mathrm{SD}$ of a range of clinically important parameters were included in the study for a more systematic comparison of the study and reference device. Among $\mathrm{FEV}_{1}, \mathrm{FVC}, \mathrm{FEV}_{6}, \mathrm{FEF}_{25-75}$ and PEF mean values for the whole study group, only the mean difference in $\mathrm{FEV}_{1}$ and $\mathrm{FVC}$ values between spirometers was found to be statistically significant $(p<0.05)$. Overall, the mean difference between spirometers was $65 \mathrm{~mL}$ for $\mathrm{FEV}_{1}$ and $59 \mathrm{~mL}$ for FVC. This is only a $2.5 \%$ difference for $\mathrm{FEV}_{1}$ and $1.8 \%$ for FVC. Although both spirometers were compliant to the ATS limits for accuracy (demonstrated in the lung simulator analysis), the statistically significant difference between the measurements for FEV 1 and FVC can be attributed to the Spirohome Clinic consistently measuring slightly higher values than the EasyOne Air (seen in the lung simulator results and regression analysis).

The mean differences of $\mathrm{FEV}_{1}$ and $\mathrm{FVC}$ between spirometers were statistically significant $(p<0.05)$ for the whole patient group, and particularly in males aged 12-18 $(p<0.05)$. Statistical significance was found in the mean differences of $\mathrm{FEV}_{6}(p<0.01)$ and PEF $(p<0.001)$ in the whole patient group and also in the male subset $\left(\mathrm{p}<0.001\right.$ for both $\mathrm{FEV}_{6}$ and PEF). Results suggest that there is influence of age and gender on $\mathrm{FEV}_{1}$ and $\mathrm{FVC}$ mean differences between spirometers. Also, the correlation between age and height also suggests that larger lung volumes in patients over the age of 12 may have attributed to the greater mean differences in several parameters recorded by each spirometer. Other demographics or anthropometrics not accounted for in this work may also have played a role in these findings.

Correlation plots for $\mathrm{FEV}_{1}$ and FVC values showed a significant ( $p<0.001$ and Pearson's correlation coefficient of 0.99$)$ agreement between spirometers for these values. The reproducibility of measurements with the Spirohome Clinic and EasyOne Air also highlighted in the Bland-Altman plots for $\mathrm{FEV}_{1}$ and $\mathrm{FVC}$ which show that most measurements are located within the lower and upper agreement limits, revealing no systematic difference between the two devices. Bland-Altman representation of age and gender subsets also reveal no systematic difference between spirometers based on these variables.

The present study demonstrates the reliability of the Spirohome Clinic in pulmonary lung function testing of respiratory patients. Fair agreement to the EasyOne Air device in both benchtop validations according to internationally accepted standards, and furthermore in clinical results strengthens the case for adopting hand-held spirometers in clinical practice. These devices have proven to be effective in pediatric patients who often find performing breathing maneuvers for spirometry a challenge, even under the guidance of a health-care professional.

Future studies building on this work should address some of the limitations of the current work. A larger data set will increase the accuracy of sample means. Similarly, a longitudinal study involving a greater number of devices will be important for examining interdevice reproducibility and holds relevance for clinical and epidemiological research. ${ }^{22}$ Although not covered in this work, the bias of chronic respiratory disease types on spirometer performance can also be explored. It will also be valuable to confirm the repeatability of the results of this study in an adult patient population.

\section{Conclusions}

In conclusion, we evaluated two portable ultrasonic spirometers currently available on the market with two complementary studies. Our findings show that the Spirohome Clinic results in statistically significant but not clinically significant higher measurements than EasyOne Air, even though both meet the ATS/ERS criteria. The precision of the Spirohome Clinic ultrasonic spirometer in clinical application is supported through the findings of this study. Ultrasonic, portable, 
durable, and user-friendly spirometers are critical tools for respiratory clinics and may continue to contribute to respiratory health by lung function measurements from the clinic to the home through teleMedicine advances.

\section{Abbreviations}

ATS, American Thoracic Society; ERS, European Respiratory Society; COVID-19, Coronavirus disease - 19; FVC, forced vital capacity; FEV1, forced expiratory volume in $1 \mathrm{~s}$; FEV6, forced expiratory volume in $6 \mathrm{~s}$; PEF, peak expiratory flow; FEF25-75, forced expiratory flow at $25-75 \%\left(\mathrm{FEF}_{25-75}\right)$ of forced vital capacity; BEV, back extrapolated volume; FET, Forced expiratory time.

\section{Acknowledgments}

The authors would like to thank Inofab Health for contributing the equipment used in this study including the spirometers and lung simulator device. The authors would also like to thank Deniz Ozdil and Dr Oya Kalaycioglu for their efforts in editing this paper and providing the statistical analyses of the data, respectively.

\section{Author Contributions}

All authors made a significant contribution to the work reported, whether that is in the conception, study design, execution, acquisition of data, analysis and interpretation, or in all these areas; took part in drafting, revising or critically reviewing the article; gave final approval of the version to be published; have agreed on the journal to which the article has been submitted; and agree to be accountable for all aspects of the work.

\section{Funding}

The authors disclosed receipt of the following financial support for the research, authorship, and/or publication of this article: Inofab Health Technologies have kindly provided the devices and test equipment used in this study and financial support for publication costs.

\section{Disclosure}

The authors have no conflicts of interest to declare.

\section{References}

1. Soriano JB, Kendrick PJ, Paulson KR, et al. Prevalence and attributable health burden of chronic respiratory diseases, 1990-2017: a systematic analysis for the Global Burden of Disease Study 2017. Lancet Respir Med. 2020;8(6):585-596. doi:10.1016/S2213-2600(20)30105-3

2. Labaki WW, Han MK. Chronic respiratory diseases: a global view. Lancet Respir Med. 2020;8(6):531-533. doi:10.1016/S2213-2600(20)30157-0

3. Fernandez-Villar A, Represas-Represas C, Mouronte-Roibas C, et al. Reliability and usefulness of spirometry performed during admission for COPD exacerbation. PLoS One. 2018;13(3):e0194983. doi:10.1371/journal.pone.0194983

4. Sears MR. Descriptive epidemiology of asthma. Lancet. 1997;350(Suppl 2):SII1-4. doi:10.1016/S0140-6736(97)90028-3

5. Beydon N, Davis SD, Lombardi E, et al. An official American Thoracic Society/European Respiratory Society statement: pulmonary function testing in preschool children. Am J Respir Crit Care Med. 2007;175(12):1304-1345. doi:10.1164/rccm.200605-642ST

6. Mycroft K, Korczynski P, Jankowski P, et al. Active screening for COPD among hospitalized smokers - a feasibility study. Ther Adv Chronic Dis. 2020;14(11):2040622320971111.

7. Korczyński P, Górska K, Jankowski P, et al. Public spirometry campaign in chronic obstructive pulmonary disease screening - hope or hype? Adv Respir Med. 2017;85(3):143-150. doi:10.5603/ARM.2017.0024

8. Avdimiretz N, Wilson D, Grasemann H. Comparison of a handheld turbine spirometer to conventional spirometry in children with cystic fibrosis. Pediatr Pulmonol. 2020;55(6):1394-1399. doi:10.1002/ppul.24743

9. Barr RG, Stemple KJ, Mesia-Vela S, et al. Reproducibility and validity of a handheld spirometer. Respir Care. 2008;53(4):433-441.

10. Mortimer KM, Fallot A, Balmes JR, Tager IB. Evaluating the use of a portable spirometer in a study of pediatric asthma. Chest. 2003;123 (6):1899-1907. doi:10.1378/chest.123.6.1899

11. Degryse J, Buffels J, Van Dijck Y, Decramer M, Nemery B. Accuracy of office spirometry performed by trained primary-care physicians using the MIR Spirobank hand-held spirometer. Respiration. 2012;83(6):543-552. doi:10.1159/000334907

12. Gerbase MW, Dupuis-Lozeron E, Schindler C, et al. Agreement between spirometers: a challenge in the follow-up of patients and populations? Respiration. 2013;85(6):505-514. doi:10.1159/000346649

13. Ramos Hernandez C, Nunez Fernandez M, Pallares Sanmartin A, et al. Validation of the portable Air-Smart Spirometer. PLoS One. 2018;13(2): e0192789. doi:10.1371/journal.pone.0192789

14. Exarchos KP, Gogali A, Sioutkou A, Chronis C, Peristeri S, Kostikas K. Validation of the portable Bluetooth(R) Air Next spirometer in patients with different respiratory diseases. Respir Res. 2020;21(1):79. doi:10.1186/s12931-020-01341-z 
15. Milanzi EB, Koppelman GH, Oldenwening M, et al. Considerations in the use of different spirometers in epidemiological studies. Environ Health. 2019;18(1):39. doi:10.1186/s12940-019-0478-2

16. Ring B, Burbank AJ, Mills K, Ivins S, Dieffenderfer J, Hernandez ML. Validation of an app-based portable spirometer in adolescents with asthma. J Asthma. 2021;58(4):497-504. doi:10.1080/02770903.2019.1702201

17. Soomer K, Claus L, Heyndrickx R, De Backer J, Oostveen E. Office spirometry: (Un)reliability of some handheld spirometers. Eur Respir J. $2013 ; 42$.

18. Carpenter DM, Jurdi R, Roberts CA, Hernandez M, Horne R, Chan A. A review of portable electronic spirometers: implications for asthma self-management. Curr Allergy Asthma Rep. 2018;18(10):53. doi:10.1007/s11882-018-0809-3

19. Tan WC, Bourbeau J, O'Donnell D, et al. Quality assurance of spirometry in a population-based study -predictors of good outcome in spirometry testing. COPD. 2014;11(2):143-151. doi:10.3109/15412555.2013.822857

20. Skloot GS, Edwards NT, Enright PL. Four-year calibration stability of the EasyOne portable spirometer. Respir Care. 2010;55(7):873-877.

21. Graham BL, Steenbruggen I, Miller MR, et al. Standardization of spirometry 2019 update. An official American Thoracic Society and European Respiratory Society technical statement. Am J Respir Crit Care Med. 2019;200(8):e70-e88. doi:10.1164/rccm.201908-1590ST

22. Kunzli N, Kuna-Dibbert B, Keidel D, et al. Longitudinal validity of spirometers-a challenge in longitudinal studies. Swiss Med Wkly. 2005;135(3334):503-508

Journal of Asthma and Allergy

Dovepress

\section{Publish your work in this journal}

The Journal of Asthma and Allergy is an international, peer-reviewed open-access journal publishing original research, reports, editorials and commentaries on the following topics: Asthma; Pulmonary physiology; Asthma related clinical health; Clinical immunology and the immunological basis of disease; Pharmacological interventions and new therapies. The manuscript management system is completely online and includes a very quick and fair peer-review system, which is all easy to use. Visit http://www.dovepress.com/testimonials.php to read real quotes from published authors.

Submit your manuscript here: https://www.dovepress.com/journal-of-asthma-and-allergy-journal 